\title{
Effects of short-term radiation emitted by WCDMA mobile phones on teenagers and adults
}

\author{
Soo Beom Choi ${ }^{1,2}$, Min Kyung Kwon ${ }^{1,2}$, Jai Won Chung ${ }^{1,3}$, Jee Soo Park ${ }^{4}$ KilSoo Chung ${ }^{5}$ and Deok Won Kim ${ }^{1,3^{*}}$
}

\begin{abstract}
Background: With the rapid increasing use of third generation (3 G) mobile phones, social concerns have arisen concerning the possible health effects of radio frequency-electromagnetic fields (RF-EMFs) emitted by wideband code division multiple access (WCDMA) mobile phones in humans. The number of people, who complain of various symptoms such as headache, dizziness, and fatigue, has also increased. Recently, the importance of researches on teenagers has been on the rise. However, very few provocation studies have examined the health effects of WCDMA mobile phone radiation on teenagers.

Methods: In this double-blind study, two volunteer groups of 26 adults and 26 teenagers were simultaneously investigated by measuring physiological changes in heart rate, respiration rate, and heart rate variability for autonomic nervous system (ANS), eight subjective symptoms, and perception of RF-EMFs during sham and real exposure sessions to verify its effects on adults and teenagers. Experiments were conducted using a dummy phone containing a WCDMA module (average power, $250 \mathrm{~mW}$ at $1950 \mathrm{MHz}$; specific absorption rate, $1.57 \mathrm{~W} / \mathrm{kg}$ ) within a headset placed on the head for $32 \mathrm{~min}$.
\end{abstract}

Results: Short-term WCDMA RF-EMFs generated no significant changes in ANS, subjective symptoms or the percentages of those who believed they were being exposed in either group.

Conclusions: Considering the analyzed physiological data, the subjective symptoms surveyed, and the percentages of those who believed they were being exposed, 32 min of RF radiation emitted by WCDMA mobile phones demonstrated no effects in either adult or teenager subjects.

Keywords: Physiological changes, Subjective symptoms, RF-EMFs perception, Provocation, ANS, Smart phones, Teenagers

\section{Background}

With the increasing use of third generation (3 G) mobile phones, social concerns have arisen concerning the possible health effects of radio frequency-electromagnetic fields (RFEMFs) emitted by wideband code division multiple access (WCDMA) mobile phones in humans [1]. On the basis of limited evidence from both human and animal studies, the World Health Organization (WHO) has classified RFEMFs as possibly carcinogenic to humans (Group 2B) [2]. WHO considered the RF-EMFs provocation studies on children of different ages to be a high-priority research in

\footnotetext{
*Correspondence: kdw@yuhs.ac

'Department of Medical Engineering, Yonsei University College of Medicine, Seoul, Republic of Korea

${ }^{3}$ Graduate Program in Biomedical Engineering, Yonsei University, Seoul, Republic of Korea

Full list of author information is available at the end of the article
}

the 2010 Research Agenda [3]. Russian National Committee on Non-Ionizing Radiation Protection (RNCNIRP) announced that absorption of EMF in a child's brain was greater than in an adult's brain because larger brain areas including those responsible for intellectual development were exposed in a child's brain in their resolution [4]. As a child's brain is also undergoing development and its intellectual functions are maturing, it is more susceptible to environmental hazards than an adult's brain.

Lindholm et al. [5] monitored local cerebral blood flow during exposure to Global System for Mobile Communication (GSM) mobile phone radiation in a teenager group (14 - 15 years old). They also measured electrocardiogram (ECG), blood pressure, and temperature simultaneously. They concluded that there were no significant changes during the short-term RF-EMFs exposure. Kramarenko 
and Tan recorded electroencephalogram (EEG) changes during the exposure of ten adults and ten children (12 years old) to a GSM phone. They suggested that cellular phones may reversibly influence the human brain [6]. Preece et al. [7] examined whether a standard mobile phone exposure at $902 \mathrm{MHz}$ had a significant effect on cognitive function in 18 children $(10-12$ years old). There was a tendency for reaction time to be shorter during exposure to radiation than in the sham condition, but no effects reached statistical significance after the Bonferroni correction. Haarala et al. [8] investigated the potential effects of a standard $902 \mathrm{MHz}$ GSM mobile phone on $10-$ 14 year old children's cognitive function, and found that the mobile phone had no effect on children's cognitive function. Kwon et al. [9] investigated the effects of GSM mobile phone use on the auditory sensory memory in 17 children (11 - 12 years old). They found that a short exposure to mobile phone EMF had no statistically significant effects on the neural change-detection profile measured with mismatch negativity. Although such studies as mentioned above have examined the effects of GSM mobile phone on teenagers or children, there are a few studies investigating about the effects of WCDMA mobile phone radiation on children or teenagers.

The autonomic nervous system (ANS) plays an important role not only in physiological situations, but also in various pathological settings. Among the different available noninvasive techniques for assessing the ANS, heart rate variability (HRV), which is obtained from heart rate, has emerged as a simple and noninvasive method to evaluate the sympathovagal balance at the sinoartrial level [10]. Respiration rate is also closely associated with HRV [11]. Therefore, we selected the three physiological variables including heart rate, respiration rate, and HRV to assess ANS activity.

In this double-blind study, two volunteer groups of 26 adults and 26 teenagers who were mostly middle school students were simultaneously investigated by measuring physiological changes in heart rate, respiration rate, and HRV for ANS, eight subjective symptoms, and perception of RF-EMFs during sham and real exposure sessions. In contrast to many other studies that have examined certain aspects of physiological changes, subjective symptoms, or perception respectively, this study investigated simultaneously these three factors to more reliably examine the bio-effects of WCDMA mobile phone radiation on two groups, especially teenagers. The aim of this study was to test whether RF-EMFs affected heart rate, respiration rate, and HRV, or gave rise to subjective symptoms in adults and teenagers. We also compared the ability of adults and teenagers to perceive exposure to RF radiation. We tested the null hypothesis that adult and teenager groups would have no differences in ANS, subjective symptoms, or perception between sham and real exposures.

\section{Methods}

\section{Participants}

The experiment was performed as a double-blind study with a total of 52 subjects: 26 adults and 26 teenagers. Only healthy subjects without any diseases and not on medications were chosen for the two groups, and 14-17 year old subjects were selected for teenager group because the experiment was demanding and potentially stressful, we did not recruit children younger than 14 years old. We used the electromagnetic hypersensitivity (EHS) screening tool developed by Eltiti et al. [12] to exclude EHS subjects. We excluded electromagnetic hypersensitive individuals, because their conditions were more psychological than physiochemical, resulting in some possible bias in our results [13]. Moreover, we already investigated effects of WCDMA mobile phone radiation on electromagnetic hypersensitive subjects [14].

As shown in Table 1, there were no significant differences in male-to-female ratio, height, weight, body-mass index, smoking status, TV viewing time per day (hr), or mobile phone usage time per day (hr) between the two groups. Because of the different characteristics of two groups, there were significant differences in age, computer usage time per day (hr), and mobile phone usage periods (yr).

The participants were advised not to consume caffeine, smoke or exercise before the day of the experiment to minimize confounding factors. All subjects, who were recruited by advertisements at the Yonsei University Health System, in Seoul, Korea, were informed of the purpose and procedure of the experiment, and were required to give written consent to participate. The Institutional Review Board of the Yonsei University Health System approved the protocol of this study (project no: 1-2010-0030).

\section{Experimental setup}

The laboratory was used exclusively for this experiment, and all other electrical devices were unplugged except for our instruments to minimize background field levels.

Table 1 Demographics of participants

\begin{tabular}{lccc}
\hline & Adult & Teenager & $P$-value \\
\hline No. of subjects $(\mathrm{n})$ & 26 & 26 & - \\
Male: female & $13: 13$ & $13: 13$ & 0.999 \\
Age $(\mathrm{yr})$ & $28.4 \pm 5.1$ & $15.3 \pm 0.7$ & $<0.001$ \\
Height $(\mathrm{cm})$ & $167.1 \pm 8.0$ & $164.4 \pm 7.3$ & 0.207 \\
Weight $(\mathrm{kg})$ & $59.4 \pm 11.1$ & $57.8 \pm 10.4$ & 0.590 \\
Body mass index $\left(\mathrm{kg} / \mathrm{m}^{2}\right)$ & $21.1 \pm 2.3$ & $21.3 \pm 2.8$ & 0.796 \\
Non-smoker: smoker & $24: 2$ & $25: 1$ & 0.999 \\
Computer usage time (hr/day) & $5.3 \pm 3.7$ & $2.2 \pm 2.0$ & 0.002 \\
TV viewing time (hr/day) & $1.7 \pm 1.1$ & $1.9 \pm 1.6$ & 0.783 \\
Mobile phone usage time (hr/day) & $0.6 \pm 0.5$ & $1.3 \pm 1.3$ & 0.116 \\
Mobile phone usage periods (yr) & $11.8 \pm 2.4$ & $5.7 \pm 1.9$ & $<0.001$ \\
\hline
\end{tabular}


Background extremely low frequency (ELF) fields at the head level in the laboratory were measured to ensure that they did not influence the subjects. The average ELF electric and magnetic fields were $1.8 \pm 0.0 \mathrm{~V} / \mathrm{m}$ and $0.02 \pm 0.01$ $\mu \mathrm{T}$, respectively, measured using an electric and magnetic field analyzer (EHP-50C; NARDA-STS, Milan, Italy). The average RF field was $0.05 \pm 0.00 \mathrm{~V} / \mathrm{m}$ with a microwave frequency range from 1920 to $1980 \mathrm{MHz}$, measured using a radiation meter (SRM 3000; NARDA-STS, Pfullingen, Germany). Both the average background ELF and RF-EMFs were negligible.

To achieve better control over exposure, we used a WCDMA module with Qualcomm chipsets (baseband: MSM6290, RF: RFR6285, power management: PM6658, San Diego, CA) to generate WCDMA RF-EMFs instead of a regular smart phone. The WCDMA module continuously transmitted at a mean output power of $250 \mathrm{~mW}(24$ $\mathrm{dBm})$ at $1950 \mathrm{MHz}$, which was measured using a wireless communication test set (E5515C, Agilent, Santa Clara, CA). The module was inserted into a dummy phone [15], and the location of the module was varied to meet the recommended restriction in specific absorption rate $(\mathrm{SAR})_{1 \mathrm{~g}}$ of $1.6 \mathrm{~W} / \mathrm{kg}$ for general public, according to the Institute of Electrical and Electronics Engineers (IEEE) Standard [16]. The SAR measurements were made with a DASY 4 measurement system (SPEAG, Zurich, Switzerland), and a Twin SAM (specific anthropomorphic mannequin) phantom was filled with head tissue-equivalent liquid (mass density, $1000 \mathrm{~kg} / \mathrm{m}^{3}$ ) as specified by the Federal Communications Commission (FCC). The measured dielectric properties of the liquid were $\sigma=1.41 \mathrm{~S} / \mathrm{m}$ and $\varepsilon_{\mathrm{r}}=39.7$ for the WCDMA frequency range. When the antenna of the module was positioned $67.5 \mathrm{~mm}$ from the ear reference point (ERP) of the dummy phone, the averaged peak spatial $\mathrm{SAR}_{1 \mathrm{~g}}$ was determined to be $1.57 \mathrm{~W} / \mathrm{kg}$ at $1950 \mathrm{MHz}$ at the left cheek position [17]. The electric field and power drift at the ERP were $6.9 \mathrm{~V} / \mathrm{m}$ and $-0.001 \mathrm{~dB}$, respectively. The measured SAR distribution is shown in Figure 1.

The module was connected via a $5 \mathrm{~m}$ USB cable and a USB type ammeter to a portable laptop computer (XNote R500, LG Electronics, Seoul, Korea), which controlled the module and monitored electrical current to check exposure conditions (Figure 2). The laptop computer was remotely controlled from another outside desktop computer to satisfy the double-blind study design. The dummy phone was attached to the subject's head using an earplug and headset to fix it at the ERP next to the cheek [18]. The phone was held at a distance of $3 \mathrm{~mm}$ from the ear using a piece of wood for insulation to prevent battery-generated heat from providing subjects with an indication that the phone was working. The apparatus was constructed from only plastic and rubber without any metal $[18,19]$.

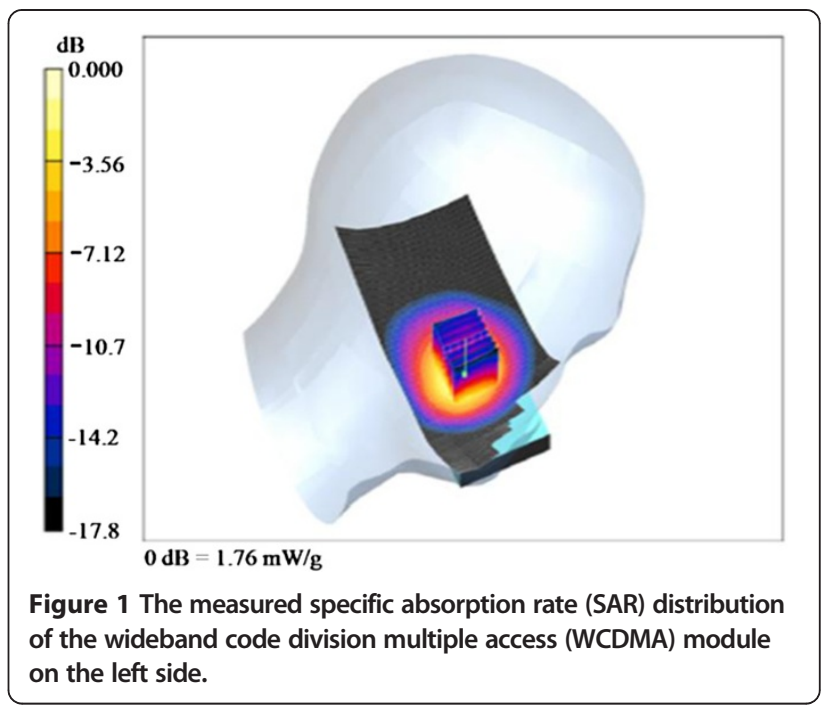

\section{Experimental procedures}

No information was given to the subjects except that they would be asked about symptoms and RF-EMFs perception at the beginning of the first experimental day. Sham and real sessions were conducted as a doubleblind test to minimize any test bias resulting from a subject and an experimenter recognizing the operational state of the WCDMA module. The experiment was performed for two days, one day for a real session and a second day for a sham session (or vice versa). No matter which came first, sham or real exposure, the second session was always conducted at approximately the same time of the day as the first session in order to maintain the subjects' physiological rhythm. The order of sham and real sessions for each subject was randomly assigned by our automatic exposure control program using MATLAB 2012b (Mathworks Inc. Natick, MA) to minimize experimental bias. The sham exposure was the first session for 14 teenagers and 15 adults. Time duration between the sessions was a minimum of one day and a maximum of 10 days.

Room temperature and relative humidity, which could considerably affect outcomes, were recorded and maintained as shown in Table 2. For the adult group, room temperature and humidity showed no significant differences between real and sham sessions. For the teenager group, room temperature and humidity showed no significant differences between real and sham sessions. For the sham sessions, room temperature and humidity showed no significant differences between adult and teenager groups. For the real sessions, room temperature and humidity showed no significant differences between adult and teenager groups.

\section{Physiological measurements}

The duration of each exposure session was $64 \mathrm{~min}$, as shown in Figure 3. Before the experiments, subjects were 


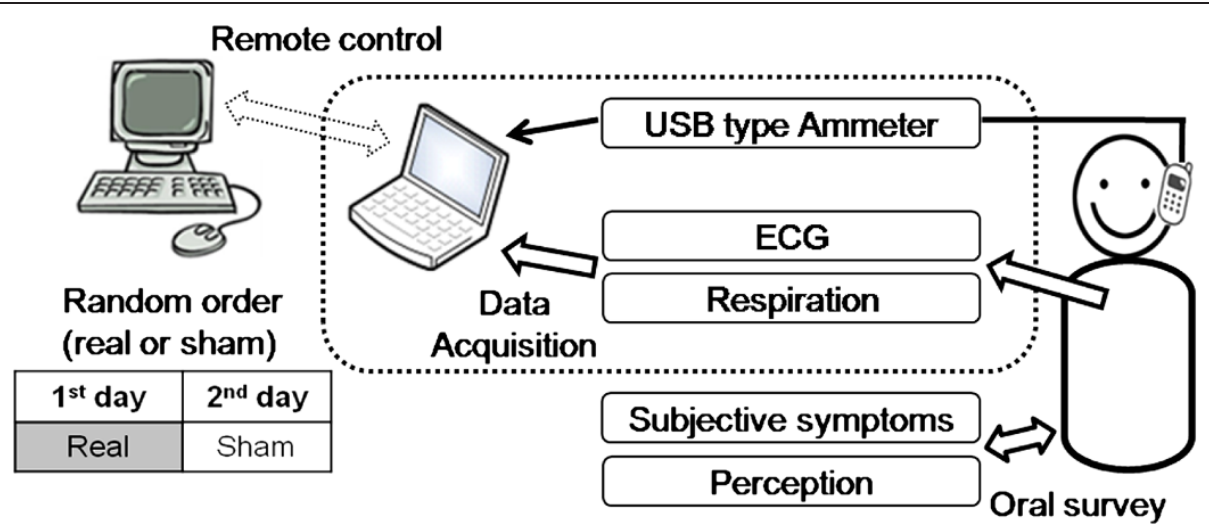

Figure 2 Block diagram of exposure setups.

instructed to rest in a sitting position for at least $10 \mathrm{~min}$. Physiological data were collected for $5 \mathrm{~min}$ each for four different stages: pre-exposure (stage I), after $11 \mathrm{~min}$ of exposure (stage II), after $27 \mathrm{~min}$ of exposure (stage III), and post-exposure (stage IV) [14]. At each stage, ECG and respiration were simultaneously measured for 5 min because of the minimum data requirement for HRV [20]. Heart rate, respiration rate, and HRV were obtained with a computerized polygraph (PolyG-I, Laxtha, Daejeon, Korea) with a sampling frequency of $512 \mathrm{~Hz}$. The data were transferred to a laptop computer (X-note R500, LG Electronics, Seoul, Korea) and analyzed using data acquisition software (Telescan 0.9, Laxtha) and analysis software (Complexity software, Laxtha). The PolyG-I recorded ECG through $\mathrm{Ag}$ - $\mathrm{AgCl}$ electrodes (2223, $3 \mathrm{M}$, St. Paul, MN) placed on both arms and the right leg of participants.

We first obtained heart rate from ECGs and then acquired HRV and the power spectrum of HRV. Highfrequency power (HFP) reflects effects on respiratory sinus arrhythmia, an index of parasympathetic nerve activity, whereas low-frequency power (LFP) reflects effects on both sympathetic and parasympathetic nerves [21]. In this study, the LFP/HFP ratio was used as an index of autonomic nerve activity balance. Respiratory inductance plethysmography, with an excitation frequency of $3 \mathrm{MHz}$, was used to measure respiration rate.

Table 2 Room temperature $\left({ }^{\circ} \mathrm{C}\right.$ ) and relative humidity (\%) in the real and sham sessions for the adult and teenager groups (mean \pm SD (min-max))

\begin{tabular}{lcccc}
\hline & Group & Real & Sham & $P$-value \\
\hline Temperature & Adult & $24.5 \pm 0.9(23-26)$ & $24.5 \pm 0.7(23-26)$ & 0.770 \\
& Teenager & $24.7 \pm 0.9(23-27)$ & $24.6 \pm 0.9(23-27)$ & 0.731 \\
& P-value & 0.430 & 0.724 & \\
Humidity & Adult & $40.5 \pm 1.9(37-45)$ & $40.3 \pm 3.2(35-52)$ & 0.823 \\
& Teenager & $41.8 \pm 2.9(38-50)$ & $41.5 \pm 2.9(38-50)$ & 0.319 \\
& P-value & 0.055 & 0.186 & \\
\hline
\end{tabular}

Subjects wore a coiled band around their upper abdomen for measurement of inductance changes resulting from cross-sectional change.

\section{Subjective symptoms and perception of RF-EMFs}

The four shaded areas in Figure 3 denote periods during which subjects were questioned about eight symptoms, with each period lasting approximately $1 \mathrm{~min}$. The eight subjective symptoms of throbbing, itching, warmth, fatigue, headache, dizziness, nausea, and palpitation were evaluated through verbal surveys, which were graded on a 4-point scale ranging from 1 (no sensation) to 4 (strong sensation) as suggested by Koivisto et al. [22]. In addition, perception of EMF exposure was investigated every $5 \mathrm{~min}$ throughout the entire session, denoted by an "o" in Figure 3. Subjects were asked to answer the question "Do you believe that you are exposed right now?" nine times during each session. Percentages of those who believed they were being exposed were calculated for pre-exposure, exposure, and post-exposure periods. The total number of inquiries was $260(5 \times 52)$ during real exposure and $676(13 \times 52)$ during nonexposure; the total number of subjects was $52(26+26)$.

\section{Data analysis}

A repeated two-way analysis of variance (ANOVA) was performed using SPSS software (SPSS 20, SPSS, Chicago, IL) to investigate differences in heart rate, respiration rate, and LFP/HFP ratio with exposure and stage for adult and teenager groups. A $P$-value $<0.05$ was considered statistically significant. Subjective symptoms, which are ordered paired data, were analyzed using a nonparametric Wilcoxon signed-rank test. A total of $64 P$ values (4 stages $\times 8$ symptoms $\times 2$ groups) were obtained for the real and sham exposure sessions for the eight symptoms at four stages in both groups. The significance level was adjusted to $0.0125(0.05 / 4)$ because testing was performed in four stages. 


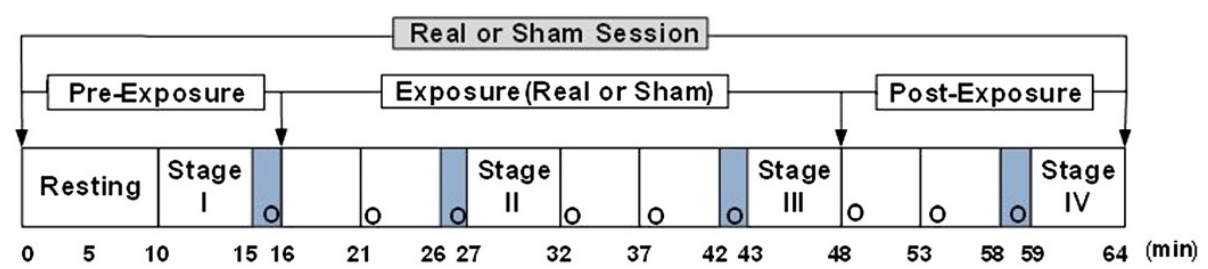

Figure 3 Experimental procedures for measuring physiological changes and investigating symptoms and perception. ECG and respiration were measured for 5 min each for four stages. The four shaded areas are periods in which the subjects were questioned about the eight symptoms. "O" indicates timing of the inquiries about RF-EMF perception during each session.

There were two exposure sessions for each participant, and nine perception inquiries for each session, as shown in Figure 3. For each session, there was one inquiry during pre-exposure, five inquiries during sham or real exposure, and three inquiries during post-exposure. In both groups, the percentages of those who believed they were being exposed were obtained and evaluated for significant differences between real and sham sessions using McNemar's test. The pre-exposure period (first inquiry) of the sham sessions was compared with that of the real sessions to test whether conditions before sham and real exposures of subjects were the same. The sham exposure period was compared with the real exposure period to test whether the subjects could detect the fields (second through sixth inquiries). The post-exposure period after sham exposure was compared with the post-exposure period after real exposure to test whether the real exposure influenced the perception of exposure in the post-exposure period (seventh through ninth inquiries).

The significance level of the exposure period was adjusted to $0.01(0.05 / 5)$, and that of the post-exposure period was adjusted to $0.017(0.05 / 3)$ because testing was for five and three inquiries. Fisher's exact test was applied to evaluate differences in the percentages of those who answered "yes", which were nominal data, between the adult and teenager groups for sham and real exposure sessions. Fisher's exact test was used because the expected values in any cells in the contingency table were below 5 .

\section{Results}

\section{Physiological variables}

Heart rate, respiration rate, and LFP/HFP ratios of the adult and teenager groups during real and sham exposures are shown in the top section of Table 3. A repeated twoway ANOVA showed no significant differences in heart rate or respiration rate for stage or exposure in either group. However, LFP/HFP ratios showed significant differences by stage in both groups, as shown in the bottom of Table 3. Therefore, a Bonferroni post hoc test was done after two-way ANOVA to investigate any differences in LFP/HFP ratios between stages for each group. For the adult group, LFP/HFP showed no significant difference between real and sham exposures $(P=0.307)$, but did show a significant difference among stages $(P=0.033)$. For the teenager group, LFP/HFP was not significantly different between real and sham exposures $(P=0.661)$, but was significantly different among stages $(P=0.002)$.

\section{Subjective symptoms and perception percentages}

Neither the adult nor the teenager group showed significant differences in any of the eight subjective symptoms surveyed (throbbing, itching, warmth, fatigue, headache, dizziness, nausea, and palpitation) between sham and real sessions in any of the four stages (Additional file 1: Table S1 and S2).

Table 4 shows the percentages of subjects who believed they were being exposed during exposure (real or sham) in the adult and teenager groups. We compared the percentages of those perceiving exposure during real and sham exposure period (second through sixth inquiries) using McNemar's test and found no significant difference between real and sham exposure period in the adult or teenager groups. To test for delayed effects of real exposure on post-exposure perception (seventh through ninth inquiries), we applied the same test and found no significant difference in the percentages of those who believed they were being exposed following real and sham exposures in the adult ( $P=0.999$ at all three inquiries) or teenager $(P=0.500, P=0.999, P=0.999)$ groups. Also, no significant difference was seen during pre-exposure period (first inquiry) between real and sham exposures in teenager $(P=0.999)$ group, indicating that the conditions experienced by subjects before real and sham exposures were the same. For adult group, we could not perform McNemar's test because no one answered "yes" in preexposure period. Similarly, a chi-square test for trend showed that the percentages of those who believed they were being exposed during pre-exposure, sham exposure, and post-exposure were not significantly different in the adult $(P=0.440)$ or teenager $(P=0.195)$ groups. This demonstrated that conditions could not be distinguished for participants throughout sham-exposure sessions.

Figure 4 shows the percentages of participants in the adult and teenager groups for each inquiry number who believed they were being exposed in sham (Figure 4A) and real (Figure 4B) exposure sessions. No significant 
Table 3 Descriptive and statistical tests for heart rate, respiration rate, and LFP/HFP ratio among stage, exposure, and interaction

\begin{tabular}{|c|c|c|c|c|c|c|c|c|c|c|c|c|}
\hline & \multicolumn{4}{|c|}{ Heart rate (bpm) } & \multicolumn{4}{|c|}{ Respiration rate (bpm) } & \multicolumn{4}{|c|}{ LFP/HFP ratio } \\
\hline & \multicolumn{2}{|c|}{ Adult } & \multicolumn{2}{|c|}{ Teenager } & \multicolumn{2}{|c|}{ Adult } & \multicolumn{2}{|c|}{ Teenager } & \multicolumn{2}{|c|}{ Adult } & \multicolumn{2}{|c|}{ Teenager } \\
\hline & Sham & Real & Sham & Real & Sham & Real & Sham & Real & Sham & Real & Sham & Real \\
\hline Stage & \multicolumn{12}{|c|}{ Mean (standard error) } \\
\hline I & $76.6(2.1)$ & $79.1(1.9)$ & $79.3(2.1)$ & $80.9(1.7)$ & $18.0(0.5)$ & $18.3(0.5)$ & $19.3(0.5)$ & $19.2(0.4)$ & $1.9(0.3)$ & $2.3(0.4)$ & $1.6(0.3)$ & $1.5(0.3)$ \\
\hline$\|$ & $76.6(2.1)$ & $77.9(1.7)$ & $79.8(1.8)$ & $80.4(1.6)$ & $18.2(0.4)$ & $18.1(0.6)$ & $19.3(0.5)$ & $19.3(0.6)$ & $2.6(0.4)$ & $3.1(0.7)$ & $1.7(0.3)$ & $2.0(0.4)$ \\
\hline III & $75.4(2.0)$ & $77.5(1.7)$ & $80.7(1.8)$ & $80.7(1.7)$ & $18.4(0.5)$ & $18.2(0.5)$ & $19.2(0.5)$ & $19.8(0.5)$ & $2.3(0.3)$ & $3.6(1.0)$ & $2.6(0.5)$ & $1.9(0.3)$ \\
\hline IV & $76.5(2.1)$ & $77.1(1.7)$ & $81.2(1.6)$ & $81.0(1.7)$ & $18.2(0.5)$ & $17.9(0.6)$ & $19.7(0.5)$ & $20.3(0.5)$ & $3.2(0.7)$ & $2.9(0.6)$ & $2.3(0.5)$ & $2.3(0.4)$ \\
\hline Factor & \multicolumn{12}{|c|}{$P$-value (F - statistic) } \\
\hline Exposure & \multicolumn{2}{|c|}{$0.328(0.997)$} & \multicolumn{2}{|c|}{$0.671(0.184)$} & \multicolumn{2}{|c|}{$0.843(0.040)$} & \multicolumn{2}{|c|}{$0.433(0.635)$} & \multicolumn{2}{|c|}{$0.307(1.088)$} & \multicolumn{2}{|c|}{$0.661(0.197)$} \\
\hline Stage & \multicolumn{2}{|c|}{$0.211(1.644)$} & \multicolumn{2}{|c|}{$0.323(1.180)$} & \multicolumn{2}{|c|}{$0.677(0.510)$} & \multicolumn{2}{|c|}{$0.067(2.481)$} & \multicolumn{2}{|c|}{$0.033^{*}(3.723)$} & \multicolumn{2}{|c|}{$0.002 *(5.492)$} \\
\hline $\begin{array}{l}\text { Interaction } \\
\text { (exposure and stage) }\end{array}$ & \multicolumn{2}{|c|}{$0.324(1.168)$} & \multicolumn{2}{|c|}{$0.209(1.600)$} & \multicolumn{2}{|c|}{$0.633(0.575)$} & \multicolumn{2}{|c|}{$0.444(0.903)$} & \multicolumn{2}{|c|}{$0.267(1.350)$} & \multicolumn{2}{|c|}{$0.222(1.562)$} \\
\hline
\end{tabular}

${ }^{*} P<0.05$, bpm; beats per min.

LFP/HFP ratio; low-frequency power/high-frequency power (power spectrum of heart rate variability).

Stage I; pre-exposure, Stage II; after $11 \mathrm{~min}$ of exposure, Stage III; after $27 \mathrm{~min}$ of exposure, Stage IV; post-exposure.

differences were seen between the adult and teenager groups in all inquiries during sham or real exposure session. Even though both groups showed low percentages of belief of being exposed during the sham exposure period (Figure 4A), they also showed low percentages during the real exposure period (Figure 4B). In summary, Table 4 shows no significant difference in perception percentages between real and sham exposure period in the adult or teenager groups. Figure 4 also shows no significant difference between the adult and teenager groups in sham or real exposure period. Therefore, we concluded that neither the adult nor the teenager group correctly perceived the RF-EMFs considering Table 4 and Figure 4.

\section{Discussion}

Neither the adults nor the teenagers showed significant differences in heart or respiration rate between real and sham exposures or among stages. For LFP/HFP, however, significant differences were seen between some stages during both real and sham exposure sessions in both groups. One disadvantage of the LFP/HFP analysis is that it is considerably influenced by stress, which can increase or decrease LFP/HFP [23]. Hjortskov et al. [24] reported that psychological stress could result in an increased LFP/HFP. Nam et al. [25] reported that LFP/HFP monotonically increased at each stage during $30 \mathrm{~min}$ of sham exposure in both EHS and non-EHS groups. In this experiment, one of the potential sources of stress was the requirement that the subjects not move during the 64-min experiment. In fact, the "no-movement" requirement was the factor that drew the most complaints from the participants, especially the teenagers. Therefore, the significant increase in LFP/HFP with time in the real and sham exposure sessions of both groups must have resulted from factors other than field exposure such as psychological stress, anxiety, or environmental factors.

For the eight subjective symptoms attributed to WCDMA mobile phone radiation, neither the adult group nor the teenager group showed significant differences between sham and real exposures in any of the four stages. Cinel et al. [26] found no evidence suggesting that exposure to mobile phone RF-EMFs affected subjective symptoms. Koivisto et al. [22] also reported that the RF-EMFs exposure did not produce any consistent subjective symptoms

Table 4 percentages of those who believed they were being exposed during sham and real exposure period, and $P$ values for sham and real exposures in adult and teenager groups

\begin{tabular}{|c|c|c|c|c|c|c|c|c|c|c|c|}
\hline \multirow[t]{3}{*}{ Group } & \multirow[t]{3}{*}{ Session } & \multicolumn{10}{|c|}{ Exposure } \\
\hline & & \multicolumn{2}{|c|}{ 2nd } & \multicolumn{2}{|c|}{$3 r d$} & \multicolumn{2}{|c|}{ 4th } & \multicolumn{2}{|c|}{5 th } & \multicolumn{2}{|c|}{ 6th } \\
\hline & & Mean (\%) & $P$-value & Mean (\%) & $P$-value & Mean (\%) & $P$-value & Mean (\%) & $P$-value & Mean (\%) & $P$-value \\
\hline \multirow[t]{2}{*}{ Adult $(n=26)$} & Sham & 7.7 & 0.999 & 7.7 & 0.999 & 3.8 & 0.999 & 7.7 & 0.999 & 3.8 & 0.999 \\
\hline & Real & 3.8 & & 3.8 & & 7.7 & & 7.7 & & 7.7 & \\
\hline \multirow[t]{2}{*}{ Teenager $(n=26)$} & Sham & 7.7 & 0.999 & 0.0 & 0.250 & 3.8 & 0.250 & 11.5 & 0.999 & 7.7 & 0.999 \\
\hline & Real & 7.7 & & 11.5 & & 15.4 & & 11.5 & & 11.5 & \\
\hline
\end{tabular}



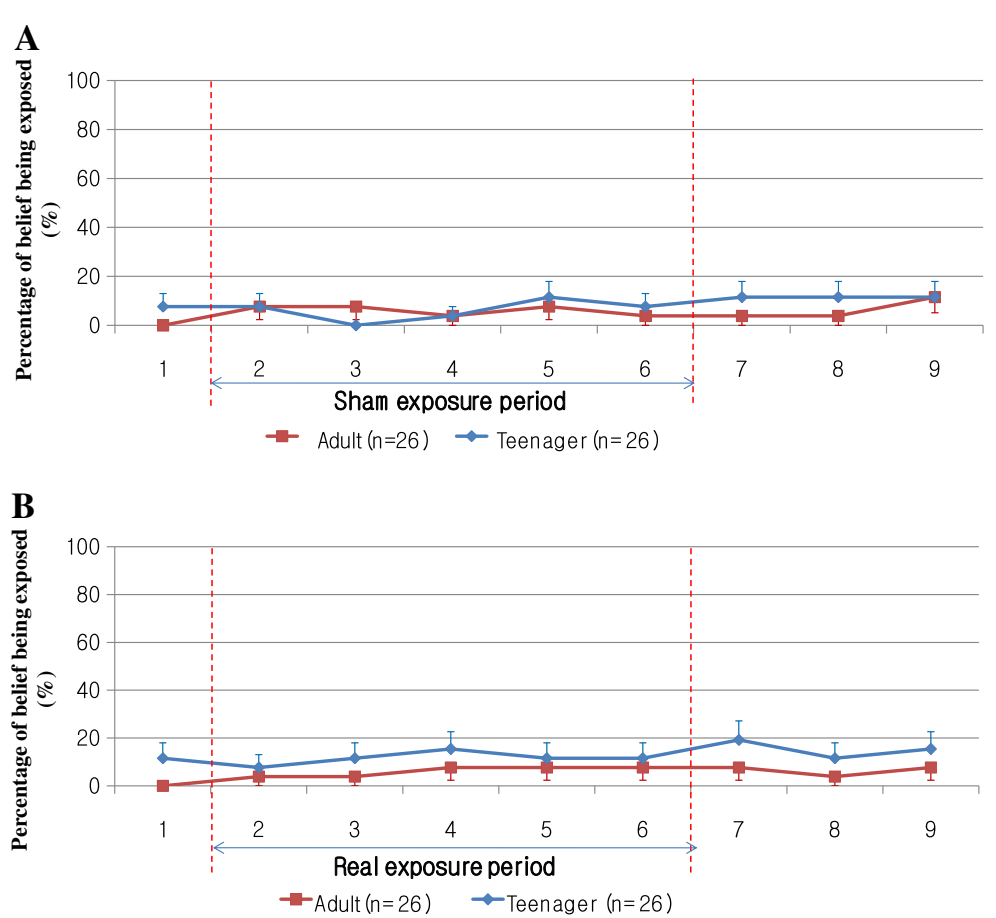

Figure 4 Percentages who believed they were being exposed at nine inquiry points in adult and teenager groups for sham (A) and real (B) exposure sessions. Bars indicate standard errors.

or sensations such as headache, dizziness, and fatigue in the non-EHS subjects. In conclusion, RF-EMFs did not give rise to subjective symptoms in adults or teenagers in this study.

No significant differences were seen in the percentages of participants who believed they were being exposed between the real and sham exposures in either the adult or the teenager group. Kwon et al. [27] reported that they found no evidence that their 84 participants perceived GSM mobile phone EMFs. All participants, even including six subjects with high self-rated sensibility, were not able to perceive mobile phone EMFs. No significant differences in percentages of perception were seen for either group among participants who believed they were being exposed during either pre-exposure or post-exposure periods between real and sham exposures. Also, no significant differences were observed in the percentages of perception for either the adult or teenager groups during sham exposure sessions (pre-exposure, sham exposure, post-exposure). Therefore, our experimental protocol appeared to be minimally biased since we confirmed no delayed effects, no differences in pre-exposure condition, and no difference in the percentages of those who believed they were being exposed during the pre-exposure, sham exposure, and postexposure periods. In this study, the subjects had only two choices, "yes" or "no", to the perception inquiry of RFEMFs. However, it could have been biased against subjects who were not sure. For future study, it is recommended to give subjects another choice, "unsure", and to exclude the answer in calculating the perception accuracy.

Children are more preferable to teenagers as participants in this study because the former are more vulnerable than the latter [28]. However, it is difficult for children due to stress to participate in our experiment, which needs a "nomovement" requirement for approximately one hour. It is also difficult to recruit children because of difficulty in obtaining parents' approval. We finally recruited teenagers as the second best. Those are the reasons why there are only a few provocation studies with children. Croft et al. [15] measured alpha activity for both GSM and WCDMA exposure among adolescents, young adults, and elderly groups. They reported an effect of GSM exposure in young adults, but observed no effect in adolescents or the elderly, or in any age group, as a function of WCDMA exposure. This result for WCDMA exposure is consistent with ours, even though they examined brain activity and we did heart rate.

There are three limitations in this study. The first limitation is the small number of participants. The number of 26 adults and 26 teenagers may not be to conclude that there are no effects of radiation emitted by WCDMA in both adults and teenagers. Moreover, any effect of WCDMA mobile phone radiation on the autonomic system might be quite limited and difficult to detect. Therefore, to draw some more definitive conclusions on this, a much larger sample will be needed. Secondly, in our study, 
more subjects received sham exposure for the first session. Ideally, the same number for each session would be better. However, the skewness is small and probably makes no difference. Lastly, we did not investigate the effects of the repetitive and daily regular exposure to RF radiation emitted by WCDMA mobile phones, which could be hazardous to teenagers, as well as adults. Therefore, further study on repetitive and daily regular exposure is necessary to examine the long-term effects, especially on teenagers.

\section{Conclusions}

In both adults and teenagers, there were no significant differences in heart rate, respiration rate, or LFP/HFP, which are all related to ANS, between sham and real exposure to a WCDMA module (average power, $24 \mathrm{dBm}$ at $1950 \mathrm{MHz}$; specific absorption rate, $1.57 \mathrm{~W} / \mathrm{kg}$ ) for $32 \mathrm{~min}$. There was no association between eight subjective symptoms and short-term RF-EMFs exposure in either group. We could not find evidences of the hypothesis that the self-perception of the exposure between two groups was different. Therefore, based on our physiological data, survey of subjective symptoms, and percentages of participants who believed they were being exposed, no effects were observed in teenagers or adults as a result of 32 min exposure to RF radiation emitted by WCDMA mobile phones.

\section{Additional file}

Additional file 1: Table S1. Eight subjective symptoms of the each stage for the real and sham sessions in the adult group. Table S2. Eight subjective symptoms of the each stage for the real and sham sessions in the teenager group.

\section{Abbreviations \\ ANOVA: Analysis of variance; ANS: Autonomic nervous system; ECG: Electrocardiogram; EEG: Electroencephalograms; EHS: Electromagnetic hypersensitivity; ELF: Extremely low frequency; EMF: Electromagnetic field; ERP: Ear reference point; FCC: Federal Communications Commission; GSM: Global System for Mobile Communications; HFP: High-frequency power; HRV: Heart rate variability; IEEE: Institute of Electrical and Electronics Engineers; LFP: Low-frequency power; max: Maximum; min: Minimum; n: Number; RF-EMFs: Radio frequency-electromagnetic fields; RNCNIRP: Russian National Committee on Non-Ionizing Radiation Protection; SAR: Specific absorption rate; SD: Standard deviation; WCDMA: Wideband code division multiple access; WHO: World Health Organization; yr: Year; 3 G: Third generation.}

\section{Competing interests}

The authors declare that they have no competing interests.

\section{Authors' contributions}

SBC set up the WCDMA module and collected experimental data. MKK performed statistical analyses, and JWC, JSP and KSC recruited the subjects and collected experimental data. DWK contributed to the development of the study protocol and editing of the manuscript. All authors read and approved the final manuscript.

\section{Acknowledgements}

This research was supported by a grant from the Basic Science Research Program through the National Research Foundation of Korea (NRF) funded by the Ministry of Education, Science and Technology (MEST) (No. 2010-0022374).

\section{Author details}

'Department of Medical Engineering, Yonsei University College of Medicine, Seoul, Republic of Korea. ${ }^{2}$ Brain Korea 21 PLUS Project for Medical Science, Yonsei University College of Medicine, Seoul, Republic of Korea. ${ }^{3}$ Graduate Program in Biomedical Engineering, Yonsei University, Seoul, Republic of Korea. ${ }^{4}$ Department of Medicine, Yonsei University College of Medicine, Seoul, Republic of Korea. ${ }^{5}$ Department of Electrical System, Dong Yang Mirae University, Seoul, Republic of Korea.

\section{Received: 10 September 2013 Accepted: 24 April 2014}

Published: 10 May 2014

\section{References}

1. Heinrich S, Thomas S, Heumann C, von Kries R, Radon K: Association between exposure to radiofrequency electromagnetic fields assessed by dosimetry and acute symptoms in children and adolescents: a population based cross-sectional study. Environ Health 2010, 9:75.

2. Baan R, Grosse Y, Lauby-Secretan B, El Ghissassi F, Bouvard V, BenbrahimTallaa L, Guha N, Islami F, Galichet L, Straif K: Carcinogenicity of radiofrequency electromagnetic fields. Lancet Oncol 2011, 12:624-626.

3. World Health Organization: WHO research agenda for radio frequency fields; 2010. [http://www.who.int/emf] Accessed August 29, 2013.

4. Grigoriev Y, Nikitina V, Grigoriev O: Resolution: Electromagnetic fields from mobile phones: Health effect on children and teenagers. Moscow: Russian National Committee on Non-lonizing Radiation Protection; 2011.

5. Lindholm H, Alanko T, Rintamäki H, Kännälä S, Toivonen T, Sistonen $H$, Tiikkaja M, Halonen J, Mäkinen T, Hietanen M: Thermal effects of mobile phone RF fields on children: a provocation study. Prog Biophys Mol Biol 2011, 107:399-403.

6. Kramarenko AV, Tan U: Effects of high-frequency electromagnetic fields on human EEG: a brain mapping study. Int J Neurosci 2003, 113:1007-1019.

7. Preece AW, Goodfellow S, Wright MG, Butler SR, Dunn EJ, Johnson Y, Manktelow TC, Wesnes K: Effect of $902 \mathrm{MHz}$ mobile phone transmission on cognitive function in children. Bioelectromagnetics 2005, 26(Suppl 7):138-143.

8. Haarala C, Bergman M, Laine M, Revonsuo A, Koivisto M, Hämäläinen H: Electromagnetic field emitted by $902 \mathrm{MHz}$ mobile phones shows no effects on children's cognitive function. Bioelectromagnetics 2005, 26(Suppl 7):144-150.

9. Kwon MS, Huotilainen M, Shestakova A, Kujala T, Näätänen $R$, Hämäläinen $H$ : No effects of mobile phone use on cortical auditory change-detection in children: an ERP study. Bioelectromagnetics 2010, 31:191-199.

10. Sztajzel J: Heart rate variability: a noninvasive electrocardiographic method to measure the autonomic nervous system. Swiss Med Wkly 2004, 134:514-522.

11. Poyhonen M, Syvaoja S, Hartikainen J, Ruokonen E, Takala J: The effect of carbon dioxide, respiratory rate and tidal volume on human heart rate variability. Acta Anaesthesiol Scand 2004, 48:93-101.

12. Eltiti S, Wallace D, Zougkou K, Russo R, Joseph S, Rasor P, Fox E: Development and evaluation of the electromagnetic hypersensitivity questionnaire. Bioelectromagnetics 2007, 28:137-151.

13. Rubin GJ, Das Munshi J, Wessely S: Electromagnetic hypersensitivity: a systematic review of provocation studies. Psychosom Med 2005, 67:224-232

14. Kwon MK, Choi JY, Kim SK, Yoo TK, Kim DW: Effects of radiation emitted by WCDMA mobile phones on electromagnetic hypersensitive subjects. Environ Health 2012, 11:69.

15. Croft RJ, Leung S, McKenzie RJ, Loughran SP, Iskra S, Hamblin DL, Cooper NR: Effects of $2 \mathrm{G}$ and $3 \mathrm{G}$ mobile phones on human alpha rhythms: Resting EEG in adolescents, young adults, and the elderly. Bioelectromagnetics 2010, 31:434-444.

16. IEEE Standard 1528-2003: Recommended Practice for Determining the Peak Spatial-Average Specific Absorption Rate (SAR) in the Human Head From Wireless Communications Devices: Measurement Techniques. IEEE Standard Coordinating Committee 34; New York; 2003 [https://ieeexplore.ieee.org/ stamp/stamp.jsp?tp=\&arnumber=1259064]

17. Beard BB, Kainz W, Onishi T, lyama T, Watanabe S, Fujiwara O, Wang J, Bit-Babik G, Faraone A, Wiart J, Christ A, Kuster N, Ae-kyoung L, Kroeze H, Siegbahn M, Keshvari J, Abrishamkar H, Simon W, Manteuffel D, Nikoloski N: Comparisons of computed mobile phone induced SAR in the SAM phantom to that in anatomically correct models of the human head. IEEE Trans Electromagn Compat 2006, 48:397-407. 
18. Haarala C, Takio F, Rintee $T$, Laine M, Koivisto M, Revonsuo A, Hamalainen H: Pulsed and continuous wave mobile phone exposure over left versus right hemisphere: effects on human cognitive function. Bioelectromagnetics 2007, 28:289-295.

19. Unterlechner M, Sauter C, Schmid G, Zeithofer J: No effect of an UMTS mobile phone-like electromagnetic field of $1.97 \mathrm{GHz}$ on human attention and reaction time. Bioelectromagnetics 2008, 29:145-153.

20. Marek MJ, Thomas BA, John C, Bobert EK, Alberto M, Arthu JM, Peter JS: Heart rate variability: standards of measurement, physiological interpretation, and clinical use. Eur Heart J 1996, 17:354-381.

21. Parazzini M, Ravazzani P, Tognola G, Thuroczy G, Molnar FB, Sacchettini A, Ardesi G, Mainardi LT: Electromagnetic fields produced by GSM cellular phones and heart rate variability. Bioelectromagnetics 2007, 28:122-129.

22. Koivisto M, Haarala C, Krause CM, Revonsuo A, Laine M, Hamalainen H: GSM phone signal does not produce subjective symptoms. Bioelectromagnetics 2001, 22:212-215.

23. Akselrod S, Gordon D, Ubel FA, Shannon DC, Berger AC, Cohen RJ: Power spectrum analysis of heart rate fluctuation: a quantitative probe of beat-to-beat cardiovascular control. Science 1981, 213:220-222.

24. Hjortskov N, Rissen D, Blangsted AK, Fallentin N, Lundberg U, Søgaard K: The effect of mental stress on heart rate variability and blood pressure during computer work. Eur J Appl Physiol 2004, 92:84-89.

25. Nam KC, Kim SW, Kim SC, Kim DW: Effects of RF exposure of teenagers and adults by CDMA cellular phones. Bioelectromagnetics 2006, 27:509-514.

26. Cinel C, Russo R, Boldini A, Fox E: Exposure to mobile phone electromagnetic fields and subjective symptoms: a double-blind study. Psychosom Med 2008, 70:345-348.

27. Kwon MS, Koivisto M, Laine M, Hamalainen H: Perception of the electromagnetic field emitted by a mobile phone. Bioelectromagnetics 2008, 29:154-159.

28. Kheifets L, Repacholi M, Saunders R, Van Deventer E: The sensitivity of children to electromagnetic fields. Pediatrics 2005, 116:e303-e313.

doi:10.1186/1471-2458-14-438

Cite this article as: Choi et al: Effects of short-term radiation emitted by WCDMA mobile phones on teenagers and adults. BMC Public Health 2014 14:438.

\section{Submit your next manuscript to BioMed Central and take full advantage of:}

- Convenient online submission

- Thorough peer review

- No space constraints or color figure charges

- Immediate publication on acceptance

- Inclusion in PubMed, CAS, Scopus and Google Scholar

- Research which is freely available for redistribution 